\title{
Signals and Learning Rules Guiding Oculomotor Plasticity
}

\author{
Soon-Lim Shin, Grace Q. Zhao, and ${ }^{\top}$ Jennifer L. Raymond \\ Department of Neurobiology, Stanford University, Stanford, California 94305-5125
}

The learning of motor skills is thought to occur largely through trial and error; however, the error signals and rules controlling the induction of motor learning have not been fully elucidated. We evaluated the learning rules that translate the sensory and motor cues available during training into learned changes in the gain and phase of the vestibulo-ocular reflex (VOR) of mice. Contrary to previous theories, neither the phase of retinal image motion relative to head motion nor the phase of retinal image motion relative to eye movement could consistently predict the direction of the learned change in the gain of the VOR across all training conditions tested. Instead, the phase of the gaze movement relative to head motion during training was the best predictor of whether learning would increase or decrease the gain of the VOR. Learned changes in the phase of the VOR were best predicted by a different cue-the phase of the eye movement relative to head motion during training. These results provide new constraints on the neural mechanisms implementing the adaptive calibration of the VOR by cerebellum-dependent motor learning.

Key words: cerebellum; eye movements; instructive signal; mice; motor learning; vestibular; VOR

\section{Introduction}

To understand the mechanisms supporting motor learning, one must determine which of the sensory and motor cues present during training provide the instructive signals guiding learning. To address this complex issue, we used a relatively simple form of motor learning, for which the relevant sensory and motor signals are readily controlled and measured. A systematic analysis of the signals guiding motor learning in simple behaviors can help to identify general principles about motor learning that would be difficult to discern for more complex behaviors. We studied a form of motor learning that modifies the eye movements driven by the vestibulo-ocular reflex (VOR). The VOR is a reflexive eye movement response to a vestibular stimulus, which stabilizes images on the retina during head motion by generating eye movements in the opposite direction from head motion. If the VOR fails to stabilize images during head motion, cerebellumdependent motor learning can adjust the gain or phase of the VOR response (Ito et al., 1974; Miles and Fuller, 1974; Gonshor and Jones, 1976; Robinson, 1976; Kramer et al., 1995; Boyden et al., 2004; Katoh et al., 2008).

Three hypotheses have been proposed about the cues controlling VOR learning. One hypothesis proposed that the cooccurrence of retinal slip and a vestibular stimulus (head motion) drives learning (Ito, 1972). A second hypothesis proposed that

\footnotetext{
Received Sept. 22, 2012; revised June 30, 2014; accepted July 1, 2014.

Author contributions:S.-L.S. and J.L.R. designed research;S.-L.S. and G.Q.Z. performed research;S.-L.S. analyzed data; S.-L.S. and J.L.R. wrote the paper.

This work was supported by NIH grants R01 NS072406, R01 DC04154, and P01 NS053862 to J.L.R. and a Katherine McCormick fellowship to S.-L.S. We thank R. Hemmati for technical assistance; and A. Katoh, R. Kimpo, T.D.B. Nguyen-Vu, A. Surrathan, 0. Winter, J. Rinaldi, and M. Goldman for helpful discussion and comments on the manuscript.

The authors declare no competing financial interests.

Correspondence should be addressed to Dr. Jennifer L. Raymond, Department of Neurobiology, Stanford University, 299 W. Campus Drive, Stanford, CA 94305-5125. E-mail: jenr@stanford.edu.

DOI:10.1523/JNEUROSCI.4510-12.2014

Copyright $\odot 2014$ the authors $\quad 0270-6474 / 14 / 3410635-10 \$ 15.00 / 0$
}

the co-occurrence of retinal slip and eye movement drives VOR learning (Collewijn and Grootendorst, 1979). A third hypothesis proposed that neurons encoding gaze velocity, in combination with vestibular signals encoding head motion, drive VOR learning (Miles and Lisberger, 1981; Lisberger et al., 1984; Shelhamer et al, 1994). Gaze velocity refers to eye movement in space, which is affected by both the eye movement relative to the head and the head motion in space.

It has been difficult to discriminate between these possibilities because head motion, retinal slip, eye movement, and gaze movement tend to covary under the conditions that are typically used to study VOR learning in the laboratory (see Fig. 1). Under such conditions, any of the three sets of cues previously hypothesized to guide VOR learning seem to be capable of determining whether learning should increase or decrease the gain of the VOR.

In the present study, we used a broad range of training paradigms to break the covariation among sensory and motor signals during VOR learning, to determine which of these signals could consistently provide the instructive signals guiding learning. We found that the direction of gaze movement relative to head motion was the best predictor of whether the gain of the VOR would increase or decrease during learning, suggesting that gaze velocity plays a key role in the induction of VOR gain learning.

We also assessed the cues driving learned changes in the phase of the VOR and found that none of the previously considered instructive signals for guiding VOR learning was adequate. Instead, the phase of the eye movements relative to head motion during training reliably predicted the direction of learned changes in the phase of the VOR.

\section{Materials and Methods}

All animal protocols were approved by the Stanford University Administrative Panel for Laboratory Animal Care. Experiments were performed on 39 adult C57BL/6J male mice (The Jackson Laboratory). Surgical methods were identical to those described previously (Boyden and Raymond, 2003). While the mouse was under anesthesia, a headpost was 
attached to the top of the skull using anchor screws and dental acrylic, and a scleral search coil (IET) was implanted on the temporal side of the right eye just beneath the conjunctiva. The search coil leads were run subcutaneously to a two-pin connector. Mice were allowed to recover from surgery for at least $5 \mathrm{~d}$ before behavioral testing.

For experiments, the head of the mouse was immobilized by attaching the implanted headpost to a restrainer. The restrainer was attached to a turntable (Carco), which delivered a vestibular stimulus by rotating the mouse about an earth-vertical axis. The restrained mouse was positioned in the center of a magnetic field generated by a set of 18 -inch magnetic coils (CNC Engineering), which was fixed to the turntable. The magnetic coils generated signals in the scleral search coil that were related to the eye position. An analog differentiator and filter with a $300 \mathrm{~Hz}$ corner frequency were used to obtain eye velocity from eye position. Gaze velocity was calculated by summing eye velocity and head velocity. All signals were digitized at a sampling frequency of $500 \mathrm{~Hz}$.

Visual motion was delivered by a moving optokinetic drum made of a white translucent plastic half-dome with black vertical stripes, each of which subtended $7.5^{\circ}$ of visual angle. The optokinetic drum was backlit by two fiber optic lights (JH Technologies). After recovery from surgery, oculomotor performance was tested on 2 consecutive days using a range of vestibular and optokinetic stimuli. A minimum of one day after the tests of oculomotor performance, motor learning was tested.

Eighteen different visual-vestibular training paradigms were used to test VOR learning. In all 18 training paradigms, the vestibular stimulus was the same, with a sinusoidal angular head velocity profile, at $1 \mathrm{~Hz}$, $\pm 10 \%$ s. This vestibular stimulus was paired with $1 \mathrm{~Hz}$ sinusoidal motion of the optokinetic drum, whose speed and phase relative to the vestibular stimulus varied across training paradigms. The 18 training paradigms included 10 gain training paradigms and 8 gainphase training paradigms.

The notation describing each training paradigm denotes the eye movement gain and phase (relative to oppositely directed head motion) that would stabilize the image of the moving visual stimulus on the retina. In each case, the ideal eye movement would be equal to (i.e., would track) the movement of the visual stimulus relative to the head. Normal viewing conditions, in which the visual stimulus is stationary in the world, require an eye movement that is equal in speed to the head motion (gain of 1 ) and exactly opposite to head motion (phase of $0^{\circ}$ ), and is hence referred to as $\times 1$. To take another example, the $\times 0.2 /+60^{\circ}$ paradigm would require a VOR with a gain of 0.2 (smaller than normal) and a phase such that eye velocity lags oppositely directed head velocity by $60^{\circ}$.

Animals were exposed to the training stimulus for six 5 min blocks ( 30 min total training). The peak speed and phase of the eye movements were measured during the first $\mathrm{min}$ and last $5 \mathrm{~min}$ of the $30 \mathrm{~min}$ training period. At the beginning of the experiment, and after each block of training, the VOR was tested in darkness with a $1 \mathrm{~Hz}, \pm 10 \%$ sinusoidal vestibular stimulus. Before the VOR was measured, a bell was rung to increase the animal's alertness, followed by an $8 \mathrm{~s}$ exposure to the vestibular stimulus in the dark before VOR measurement commenced.

Thirty-nine mice were tested on multiple training paradigms. To allow the VOR gain to return to baseline between experiments, mice were placed in their home cages in a normal visual-vestibular environment for at least $48 \mathrm{~h}$ after an increase in VOR gain, and at least $72 \mathrm{~h}$ after a decrease in VOR gain (Kimpo et al., 2005). The order of the training paradigms was randomized in each mouse.

The eye movement, gaze, and retinal slip trajectories during training were characterized by their peak speed and phase relative to head motion or each other. All analyses were performed off-line using MATLAB (MathWorks) and Microsoft Excel (Microsoft). Multiple cycles of head and eye velocity were aligned on the zero crossings of head velocity and then averaged. Any cycle containing a saccade or motion artifact was excluded. The amplitude and phase of the eye movement were extracted from a sinusoidal fit to the averaged eye movement. Retinal slip was calculated as the difference between the visual stimulus motion and the sinusoidally fit eye movement.

The VOR gain was calculated as the ratio of the eye speed to head speed, measured in darkness. The VOR phase was calculated as the difference between the eye velocity phase and the head velocity phase in the opposite direction. A perfectly compensatory VOR would thus have a phase of zero. Learned changes in VOR gain were measured as the percentage change in VOR gain measured immediately after 30 min of training, compared with the initial VOR gain measured immediately before training. Learned changes in VOR phase were measured by subtracting the phase before training from the phase after training.

The significance of learned changes in the VOR induced by each training paradigm was determined by comparing the gain and phase of the VOR measured in the dark before and after the training using a paired Student's $t$ test with Bonferroni correction for multiple comparisons.

To quantify the ability of the candidate instructive signals during training to predict the direction of the learned change in VOR gain or phase, we calculated the mutual information as follows:

$$
\sum_{x} \sum_{y} p(x, y) \log _{2} \frac{p(x, y)}{p(x) p(y)}
$$

where $p(x)$ is the probability of a particular learning outcome, $p(y)$ is the probability of a candidate instructive signal taking a particular value during training, and $p(x, y)$ is the joint probability. There were three possible learning outcomes that were determined by a paired Student's $t$ test with Bonferroni correction; $x \in$ \{significant decrease in VOR gain, no significant change in VOR gain, significant increase in VOR gain $\}$. The same value was assigned to all individual experiments with a given training paradigm. To compute the information carried by candidate error signals R-H, R-E, G-H, E-H, and R-G, the variable $y$ was a phase in the range $0 \sim 2 \pi$, using bins of $1,2,5,10$, or $20^{\circ}$. $p(y)$ was computed from the phase values from each individual experiment (each mouse on each training paradigm). To compute the information carried by candidate error signals $R_{s}, E_{s}$, or $G_{s}$, the variable $y$ was a peak speed in the range $0 \sim 18 \%$, with bins of $0.1,0.2,0.5,1$, or $2 \%$ s. $p(y)$ was computed from the peak speeds in each individual experiment.

\section{Results}

VOR learning is induced by pairing a vestibular stimulus with a moving visual stimulus. Hence, the sensory and motor signals present during training that are most likely to control the induction of VOR learning are those encoding the vestibular stimulus (head motion), image motion on the retina (retinal slip), and/or correlates of these signals, such as eye movement or gaze movement. We designed a set of training paradigms that elicited different combinations of these signals in mice. We then evaluated whether the three candidate instructive signals proposed by previous studies, the direction (phase) of retinal slip relative to head motion (R-H), the direction (phase) of retinal slip relative to eye movement (R-E), or the direction (phase) of gaze movement relative to head motion $(\mathrm{G}-\mathrm{H})$, were consistently correlated with the learned change in VOR gain, and might therefore be driving the learning.

Each training paradigm paired a vestibular stimulus with visual stimulus motion. The vestibular stimulus consisted of passive, sinusoidal rotation about an earth-vertical axis at $1 \mathrm{~Hz}$, with peak velocity of $10^{\circ} \mathrm{s}$. The visual stimulus was provided by sinusoidal rotation of an optokinetic drum about the same axis. In different training paradigms, we varied the speed and phase of the visual stimulus motion relative to the vestibular stimulus, which induced a broad range of learned changes in VOR gain and phase.

First, we tested a series of 10 "gain training" paradigms, denoted $\times 0, \times 0.2, \times 0.3, \times 0.6, \times 0.8, \times 1, \times 1.2, \times 1.4, \times 1.6$, and $\times 2$, where the notation " $\mathrm{x} a$ " refers to a training stimulus that required a tracking eye movement gain of " $a$ " relative to head motion to stabilize the visual stimulus on the retina. Across the 10 gain training paradigms, there was considerable covariation between candidate instructive signals (Fig. 1, black circles). To break the covariation, we used a set of eight additional, "gainphase training" paradigms, denoted $\times 1 /-90^{\circ}, \times 0.2 /-60^{\circ}$, 
A

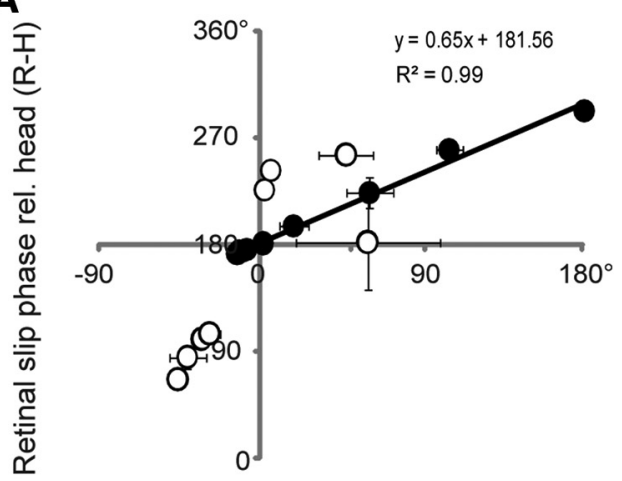

Retinal slip phase rel. eye (R-E)

B

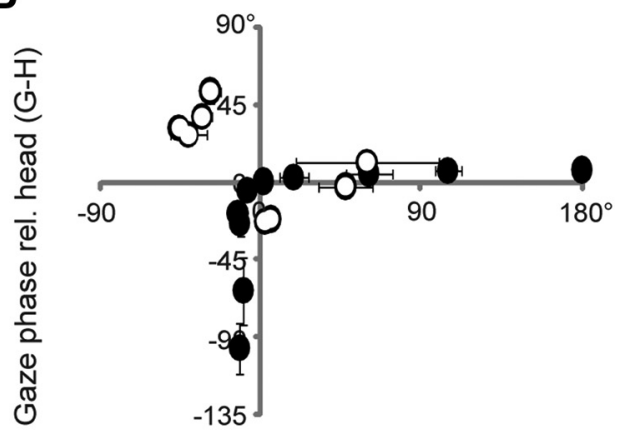

Retinal slip phase rel. eye (R-E)

\section{C}

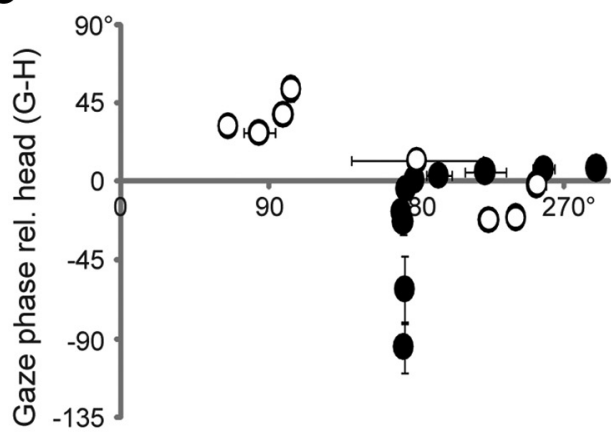

Retinal slip phase rel. head (R-H)

Figure 1. Covariation between putative instructive signals present during VOR training. $\boldsymbol{A}$, During the VOR gain training paradigms (black circles, solid line), the phase of retinal slip relative to head motion $(\mathrm{R}-\mathrm{H})$ was highly correlated with the phase of retinal slip relative to eye movement (R-E), but the correlation was weaker during gain-phase training (white circles). $\boldsymbol{B}$, C, The phase of gaze movement relative to head motion $(\mathrm{G}-\mathrm{H})$, plotted relative to $\mathrm{R}-\mathrm{H}$ or $\mathrm{R}-\mathrm{E}$ during training.

$\times 0.6 /-60^{\circ}, \times 1 /-60^{\circ}, \times 1.6 /-60^{\circ}, \times 1 / 45^{\circ}, \times 0.2 / 60^{\circ}$, and $\times 1 /$ $60^{\circ}$, where the notation " $\times a / b^{\circ}$ " refers to a training stimulus that required an eye movement with gain "a" and phase "b" relative to oppositely directed head motion to stabilize the visual stimulus on the retina. Positive values indicate a lag relative to oppositely directed head velocity. The gain-phase training paradigms reduced the covariation between the candidate instructive signals (Fig. 1, white circles).

Therefore, we assessed which candidate instructive signal could better predict VOR learning across all 18 training paradigms: the phase of retinal slip velocity relative to head velocity (R-H), the phase of retinal slip velocity relative to eye velocity
(R-E), or the phase of gaze velocity relative to head velocity (G$\mathrm{H}$ ). We also assessed five additional candidate instructive signals: the phase of eye velocity relative to head velocity $(\mathrm{E}-\mathrm{H})$, the phase of retinal slip relative to gaze movement (R-G), the peak retinal slip speed $\left(R_{s}\right)$, the peak eye speed $\left(E_{s}\right)$, and the peak gaze speed $\left(\mathrm{G}_{\mathrm{s}}\right)$.

The direction of retinal slip relative to head motion does not reliably predict the direction of learned changes in VOR gain One learning rule that has been proposed for the VOR is that the direction of retinal slip relative to head motion controls the direction of the learned change in VOR gain (Ito, 1972). In particular, it has been proposed that retinal slip in the opposite direction from head motion would indicate the need for a bigger VOR response and induce an increase in VOR gain, whereas retinal slip in the same direction as head motion would signal the need for and induce a decrease in VOR gain. Our results were consistent with this candidate learning rule, if we only considered the instructive signals available during two of the most commonly used training paradigms: one that induced a learned decrease in VOR gain and one that induced a learned increase in VOR gain. In particular, we compared the instructive signals present during $\times 0$ training, in which the visual stimulus moves exactly with the head during training and induces a learned decrease in VOR gain, with the instructive signals present during $\times 2$ training, in which the visual stimulus moves at the same speed as the head but in the opposite direction, and induces a learned increase in VOR gain (Fig. 2). The results from the population of mice tested were tightly clustered. During $\times 2$ training, peak retinal slip was closely aligned with peak head motion in the opposite direction (Fig. 2E,F, middle, red histogram aligned with black bar centered on $180^{\circ}$ ), but during $\times 0$ training, peak retinal slip was better aligned with peak head motion in the same direction (Fig. 2E, F, left, blue histogram aligned with white bar). Thus, the direction, or phase, of retinal slip relative to peak head motion was different during $\times 0$ training versus $\times 2$ training, consistent with the hypothesis that the direction of retinal slip relative to head motion discriminates the need for an decrease versus increase in VOR gain, and controls the different learned changes in VOR gain induced by these two training paradigms.

However, this rule failed to account for the learned changes in VOR gain when we tested a broader range of training paradigms. For example, during $\times 0.6$ training, retinal slip was aligned with head motion in the opposite direction (Fig. $2 E, F$, right, blue histogram aligned with black bar) as it was during $\times 2$ training, although $\times 0.6$ training induced a learned decrease in VOR gain, whereas $\times 2$ training induced an increase in VOR gain (Fig. $2 I$ ). More generally, when the full range of 10 gain training paradigms was considered, it was possible for a decrease, increase, or no change in VOR gain to be induced when retinal slip was closely aligned with head motion in the opposite direction during training (Fig. $3 A_{1}, B_{1}$, blue, red, and black histograms of $\mathrm{R}-\mathrm{H}$ values near $180^{\circ}$ ).

Across the set of 10 gain training paradigms, there was a small but consistent difference between the phase of retinal slip relative to head motion $(\mathrm{R}-\mathrm{H})$ during training paradigms that induced a decrease in VOR gain (Fig. $3 A_{1}, B_{1}$, blue histograms and shading; retinal slip lagged oppositely directed head motion by at least a few degrees) versus those that induced no change or an increase in VOR gain (Fig. $3 A_{1}, B_{1}$, black and red histograms and pink shading). However, this pattern did not hold across the eight additional gain-phase training paradigms (Fig. $3 A_{2}, B_{2}$ ). In partic- 
$x 0$

A Visual stimulus

B

Head motion

C Eye movement

D Eye movement phase

G

E Retinal slip F $\underset{\text { Retinal slip }}{\text { phase }}$

H Gaze
phase $\Delta$ VOR Gain (\%)
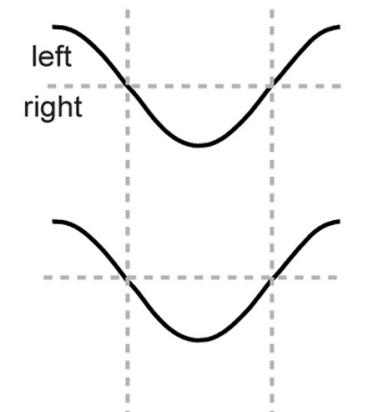

[

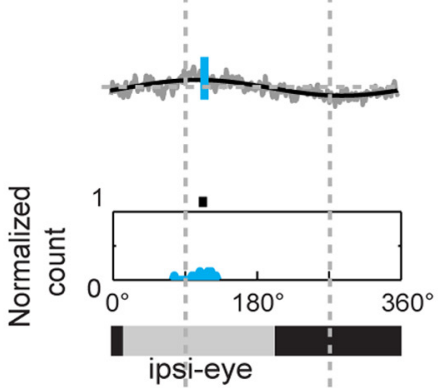

$\left[\begin{array}{l}\mathrm{O} \\ \frac{\mathrm{N}}{\mathrm{T}} \\ \mathrm{E}\end{array}\right.$

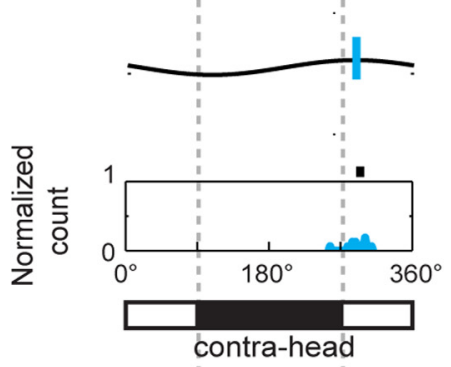

contra-head
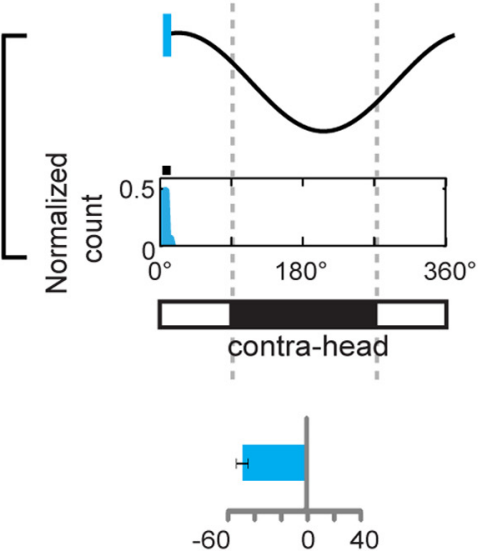

$x 2$

x0.6
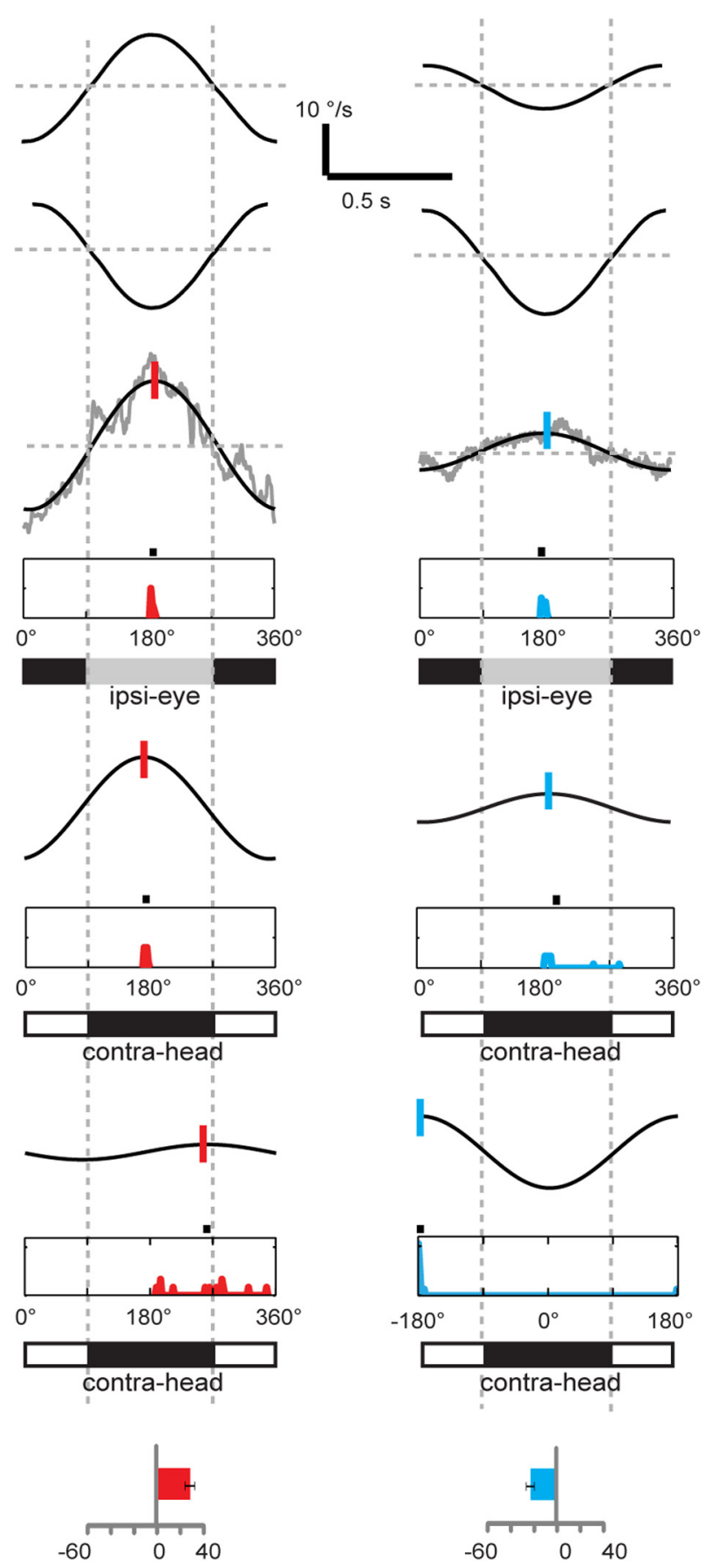

Figure 2. Signals present during $\times 0$ (left), $\times 2$ (middle), and $\times 0.6$ (right) training. $A$, Angular velocity of the visual stimulus about an earth-vertical axis. $B$, Angular velocity of the head. $C$, Example traces showing the eye velocity in one representative animal. Superimposed tic marks indicate the phase of the eye movement responses. $\boldsymbol{D}$, Normalized histograms showing the phase of the eye movement responses across the population of animals. Black tic marks indicate the mean of each distribution; gray bars represent the half of the stimulus cycle centered on this value (Ipsi-eye). $\boldsymbol{E}$, Retinal slip in the representative experiment shown in $\boldsymbol{C}$. Retinal slip was calculated as the difference between the visual stimulus motion and the sinusoidally fitted eye movement. $\boldsymbol{F}$, Normalized histograms showing the phase of retinal slip across the population of animals. Black tic marks indicate the mean of each distribution. Black and white bars represent the alignment of retinal slip with head motion in the opposite direction (black bars, contra-head) or same direction (white bars, ipsi-head), respectively. G, Gaze velocity in the representative experiment shown in C. $H$, Normalized histograms showing the phase of gaze movements across the population of animals. I, Learned change in VOR gain induced by 30 min of training (mean \pm SEM). Blue represents results from training stimuli that induce a learned decrease in VOR gain; red represents results from training stimuli that induce a learned increase in VOR gain.

ular, some training paradigms with retinal slip lagging oppositely directed head motion induced no learning (Fig. $3 A_{2}, B_{2}, \times 1 / 60^{\circ}$ ) or even a trend for an increase in VOR gain (Fig. $3 A_{2}, B_{2}$, black arrow, $\times 1 / 45^{\circ}$ ), rather than the decrease in VOR gain that would be predicted based on the gain-training paradigms (Fig. $3 B_{1}, B_{2}$, blue shading). Moreover, during some training paradigms, such as $\times 0.2 /-60^{\circ}$, the retinal slip amplitude was low; hence, the phase of the retinal slip was highly variable across different animals undergoing the same training, even though the learning outcome was highly consistent across animals (Fig. $3 A_{2}, B_{2}$ ). 


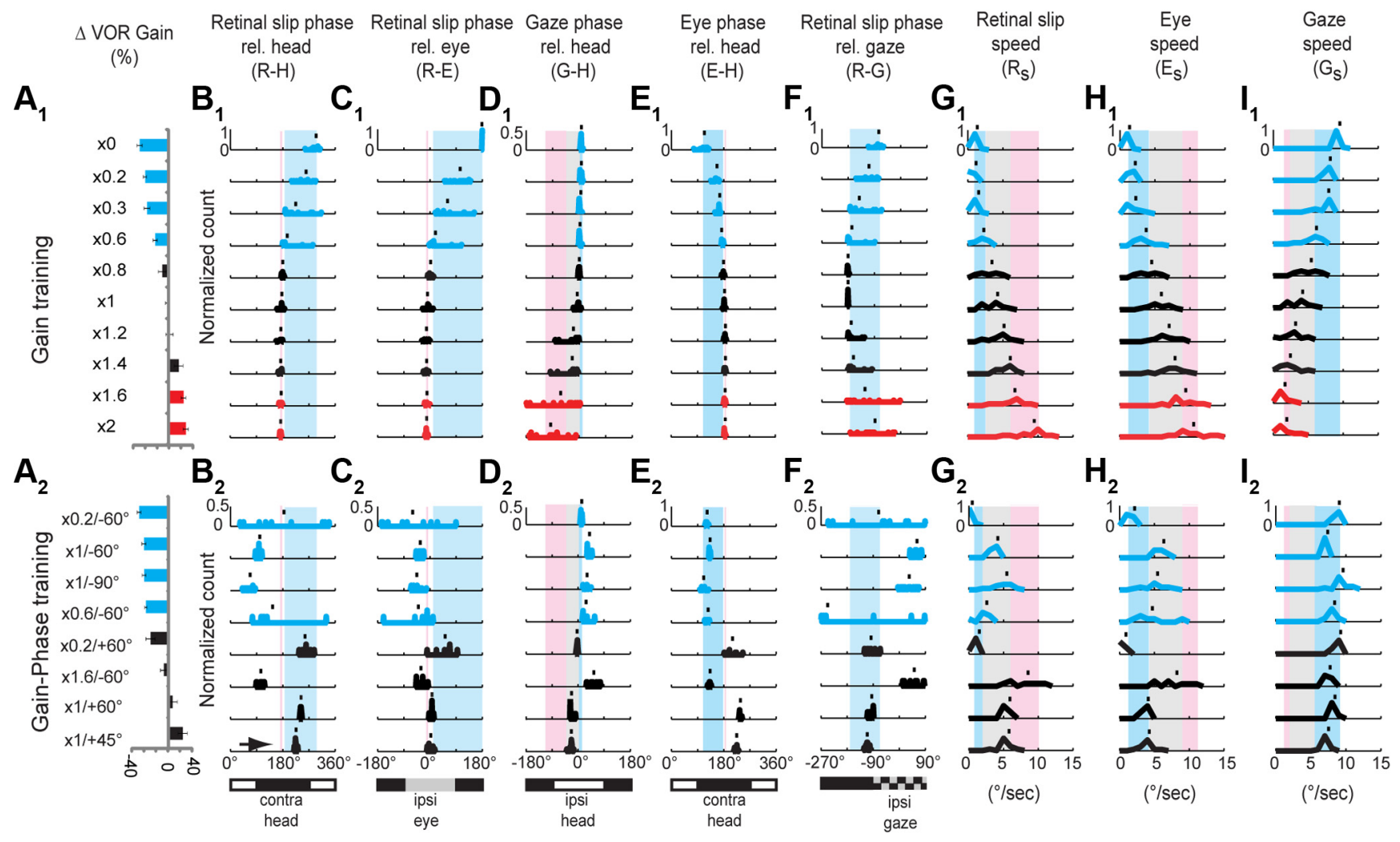

Figure 3. Learned changes in VOR gain induced by a broad range of VOR gain training paradigms $\left(\boldsymbol{A}_{7}\right)$ and gain-phase training paradigms $\left(\boldsymbol{A}_{2}\right)$, and the candidate instructive signals present during training $(\boldsymbol{B}-\boldsymbol{I}) . \boldsymbol{A}_{1}, \boldsymbol{A}_{2}$, Learned change in VOR gain after 30 min training. Blue (red) bars represent a significant decrease (increase) in VOR gain ( $p<0.05$, Bonferroni-corrected $t$ test). Black represents no significant learned change in VOR gain. This color code is used throughout all figures. $\boldsymbol{B}_{1}, \boldsymbol{B}_{2}$, Phase of retinal slip relative to head motion ( $\left.\mathrm{R}-\mathrm{H}\right)$ during training. Normalized histograms represent the distributions for the population of animals tested; $180^{\circ}$ indicates retinal slip in phase with head motion in the opposite direction (Contra-head), with increasing values representing increasing phase lag. $C_{1}, C_{2}$, Normalized histograms showing the phase of retinal slip relative to eye movement during training $(\mathrm{R}-\mathrm{E}) ; 0^{\circ}$ indicates retinal slip in phase with eye movement in the same direction. $\boldsymbol{D}_{1}, \boldsymbol{D}_{2}$, Phase of gaze relative to head motion during training $(\mathrm{G}-\mathrm{H}) . \boldsymbol{E}_{1}, \boldsymbol{E}_{2}$, Phase of eye movement relative to head motion during training $(\mathrm{E}-\mathrm{H}) . \boldsymbol{F}_{1}, \boldsymbol{F}_{2}$, Phase of retinal slip relative to gaze movement during training (R-G). $G_{1}, G_{2}$, Normalized histograms showing peak speed of retinal slip during training $\left(R_{s}\right) . H_{1}, H_{2}$, Peak speed of eye movement during training $\left(E_{s}\right) . I_{1}, \boldsymbol{I}_{2}$, Peak speed of gaze during training $\left(G_{s}\right)$. Blue and pink shading represents range of parameters associated with a learned decrease or increase in VOR gain, respectively, during the gain training paradigms shown in $A_{1}-I_{1}$. Light gray shading represents range of parameters associated with no significant gain change. No shading represents parameter space not sampled during gain training. Range of parameters shaded was determined by mean \pm SEM of training paradigms at the border. Tic marks above each histogram indicate the mean.

Moreover, the phase of $\mathrm{R}-\mathrm{H}$ did not discriminate training paradigms that induced an increase in VOR gain versus no change in VOR gain (Fig. $3 B_{1}$, black and red histograms) and therefore does not appear to be the instructive signal controlling whether learning induces an increase versus no change in VOR gain. For example, the phase of retinal slip relative to head motion was very similar during $\times 1.2$ and $\times 2$ training (Fig. $3 B_{1}, p>$ 0.3 ), but $\times 1.2$ training induced no learning, whereas $\times 2$ training induced a significant increase in VOR gain (Fig. $3 A_{1}$ ). Therefore, the phase, or timing, of retinal slip relative to head motion $(\mathrm{R}-\mathrm{H})$ during training cannot be the instructive signal that determines the direction of VOR learning across the full range of training stimuli.

\section{The phase of retinal slip relative to eye movement does not reliably predict the direction of the learned change in VOR gain}

A second learning rule that has been proposed for the VOR is that the direction of retinal slip relative to eye movement, rather than head motion, controls the direction of the learned change in VOR gain (Collewijn and Grootendorst, 1979). In particular, if retinal slip is in the same direction as the eye movement, then that would indicate that the eye movement needs to be bigger, and the gain of the VOR should increase; but if retinal slip is in the opposite direction from the eye movement, then that would indicate that the eye movement is too big, and the gain of the VOR should decrease. Accordingly, we observed retinal slip in the same direction as eye movement during $\times 2$ training (red retinal slip histogram in Fig. 2E, F, center aligned with upward deflection of eye movement trace in Fig. $2 C$, center and gray bar indicating ipsiversive eye movement in Fig. $2 D$, middle), but in the opposite direction to the eye movement during $\times 0$ training (blue retinal slip histogram in Fig. $2 E$, F, left aligned with downward deflection of eye movement trace in Fig. 2C, left and black bar indicating contraversive eye movement in Fig. 2D, left), as reported in previous studies. Thus, the direction of retinal slip relative to the eye movement discriminated the training paradigm that induced an increase versus decrease in VOR gain and thus seemed to carry the information required to trigger the different changes in behavior that are induced by these two training paradigms (Fig. 2I).

However, when the full range of training paradigms was considered, the direction of the learned change in VOR gain could not be predicted from the direction of retinal slip relative to the eye movement. For example, during $\times 0.6$ training, retinal slip was in the same direction as eye movement (blue retinal slip histogram in Fig. $2 E, F$, right aligned with gray bar in Fig. $2 D$, right), as it was during $\times 2$ training, although $\times 0.6$ training induced a learned decrease in VOR gain, whereas $\times 2$ training in- 
duced an increase in VOR gain (Fig. 2I). More generally, it was possible for either a decrease in VOR gain, increase in VOR gain, or no change in VOR gain to be induced when retinal slip was closely aligned with eye movement in the same direction (Fig. $3 C_{1}, C_{2}$, blue, red, and black histograms of R-E values close to $0^{\circ}$ ).

Across the set of 10 gain training paradigms, the phase of R-E during training paradigms that induced a significant decrease in VOR gain (Fig. $3 C_{1}$, blue shading) lagged the phase of R-E during training paradigms that induced no change or a learned increase in VOR gain (Fig. $3 C_{1}$, pink shading). However, when the gainphase training paradigms were also considered, there were several violations of this pattern. For example, a training paradigm that induced a decrease in VOR gain $\left(\times 0.6 /-60^{\circ}\right)$ had an R-E distribution highly overlapping with those of training paradigms that increase VOR gain (Fig. $2 C_{2}$, blue histogram overlapping pink shading). Moreover, R-E was similar during training paradigms that induced no change versus an increase in VOR gain (Fig. $3 C_{1}, C_{2}$, black vs red histograms). Thus, the learned change in VOR gain could not be reliably predicted from the phase of retinal slip relative to eye movement during training (R-E).

\section{The phase of gaze velocity relative to head velocity reliably predicts the direction of the learned change in VOR gain} A third learning rule that has been proposed for the VOR is that gaze velocity, in combination with vestibular signals, controls the direction of the learned change in VOR gain (Miles and Lisberger, 1981; Lisberger et al., 1984; Lisberger and Pavelko, 1986; Shelhamer et al., 1994). During $\times 0$ training, gaze velocity was aligned with ipsiversive head velocity (Fig. $2 \mathrm{H}$, left, blue histogram), whereas during $\times 2$ training, gaze velocity led ipsiversive head velocity, by $\sim 90^{\circ}$ on average (Fig. $2 \mathrm{H}$, middle, red histogram). During $\times 0.6$ training, gaze velocity was aligned with ipsiversive head velocity (Fig. $2 \mathrm{H}$, right, red histogram), as it was during $\times 0$ training, consistent with the observation that both training paradigms induced a learned decrease in VOR gain (Fig. $2 I)$. Moreover, when the full range of training paradigms was considered, the range of $\mathrm{G}-\mathrm{H}$ values during the gain-decrease training paradigms (Fig. $3 D_{1}, D_{2}$, blue shading) was separated from the $\mathrm{G}-\mathrm{H}$ values during training paradigms that either induced a VOR gain increase or no change (Fig. $3 D_{1}, D_{2}$, pink shading). Gaze slightly lagged ipsiversive head motion during training paradigms that induced a learned decrease in VOR gain but led ipsiversive head motion during training paradigms that induced a learned increase in VOR gain. G-H had intermediate values during the training paradigms that induced no change in VOR gain (Fig. $3 D_{1}, D_{2}$, gray shading), with one exception, $\times 1.6 /-60^{\circ}$. During $\times 1.6 /-60^{\circ}$ training, gaze lagged head motion by more than it did during training paradigms that induced a decrease in VOR gain; hence, it may have exceeded the effective range for inducing a learned decrease in VOR gain.

The G-H values during training predicted not only the mean learned change in VOR gain across training paradigms, but also the changes in VOR gain observed in individual experiments. There was a consistent correlation between the G-H values measured during a given training session and the learned change in VOR gain induced in that session (Fig. 4A).

\section{Other candidate instructive signals}

We considered five additional candidate instructive signals: the phase of eye movement relative to head motion $(\mathrm{E}-\mathrm{H})$, the phase of retinal slip relative to gaze (R-G), the peak speed of retinal slip $\left(R_{s}\right)$, the peak speed of eye movement $\left(E_{s}\right)$, and the peak speed of

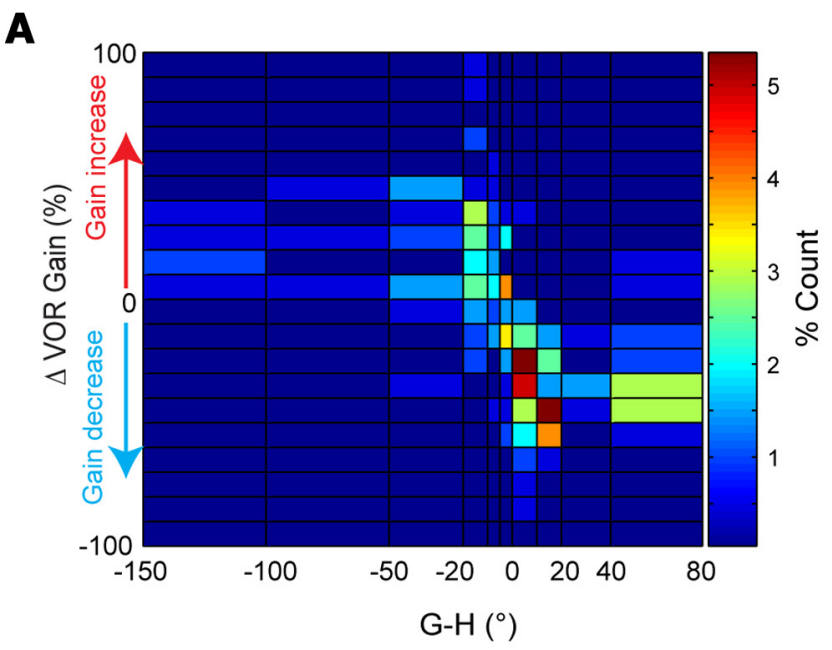

B

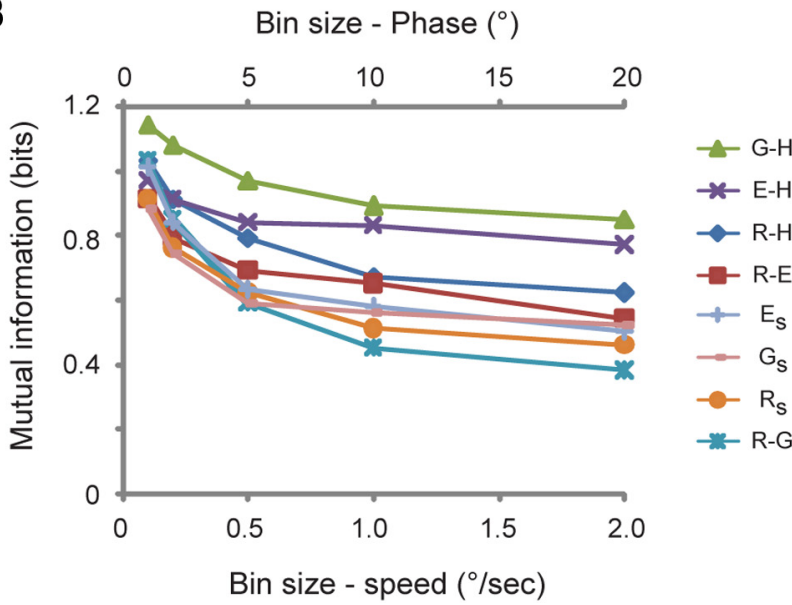

Figure 4. Gaze relative to head $(\mathrm{G}-\mathrm{H})$ was the best predictor of learned change of VOR gain $\boldsymbol{A}$, Joint histogram showing the correlation between $\mathrm{G}-\mathrm{H}$ and the learned change of VOR gain across individual training sessions. $\boldsymbol{B}$, Mutual information about the learned gain change carried by the phase of gaze relative to head $(\mathrm{G}-\mathrm{H})$ and other candidate instructive signals. Mutual information was calculated in bin sizes of $1,2,5,10$, or $20^{\circ}$ for $\mathrm{G}-\mathrm{H}, \mathrm{E}-\mathrm{H}, \mathrm{R}-\mathrm{H}$, and $\mathrm{R}-\mathrm{G}$ and bin sizes of $0.1,0.2,0.5,1$, or $2 \%$ for $R_{s}, E_{s}$, or $G_{s}$.

gaze movement $\left(G_{s}\right)$. None of these could reliably predict the direction of the learned change in VOR gain induced by the different training paradigms.

There was less variation in E-H across training paradigms that induced different changes in VOR gain (Fig. $3 E_{1}, E_{2}$ ) than the variation in $\mathrm{R}-\mathrm{H}, \mathrm{R}-\mathrm{E}$, or $\mathrm{G}-\mathrm{H}$, especially for the gain training paradigms; hence, E-H would be a less robust instructive signal. The direction of the learned change in VOR gain could not be predicted from R-G because the range of R-G values during training paradigms that induced a learned decrease in VOR gain (Fig. $3 F_{1}, F_{2}$, blue shading and histograms) encompassed the range of $R-G$ values during training paradigms that induced no change in VOR gain (Fig. 3, black histograms) or a gain increase (Fig. 3, red histograms). Likewise, the speed of retinal slip $\left(\mathrm{R}_{\mathrm{s}}\right)$, of the eye movements $\left(\mathrm{E}_{\mathrm{s}}\right)$, and of the gaze movements $\left(\mathrm{G}_{\mathrm{s}}\right)$ during training were not consistently predictive of the occurrence and direction of the learned change in VOR gain, when all training paradigms were considered (Fig. 3G-I).

Thus, on average, of the eight candidate instructive signals assessed, the phase of gaze relative to head motion during training $(\mathrm{G}-\mathrm{H})$ appeared to be the best predictor of the learned change 


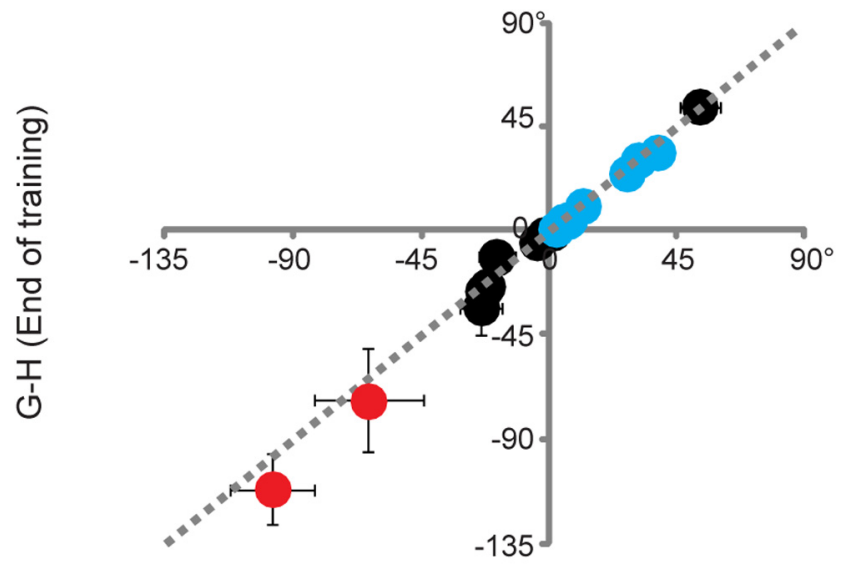

G-H (Begining of training)

Figure 5. Consistent phase of gaze relative to head (G-H) throughout the training period. $\mathrm{G}-\mathrm{H}$ during the last 5 min of training ( $y$-axis) was similar to that during the first min of training ( $x$-axis). Each point represents the results from one of the 18 training paradigms. Dotted line indicates diagonal. Blue (red) points represent results from training paradigms that induced a significant decrease (increase) in VOR gain ( $p<0.05$, Bonferroni-corrected $t$ test). Black represents no significant learned change in VOR gain.

in VOR gain. To quantify this, we calculated the information carried by each of the eight candidate instructive signals about whether a training paradigm would induce a significant learned increase in VOR gain, a significant learned decrease in VOR gain, or no significant change in VOR gain (Fig. 4B; see Materials and Methods). The calculation of mutual information considers the variability across both individual animals and across training paradigms.

This calculation of mutual information confirmed that G-H carried more information about the change in VOR gain induced by each training paradigm than any of the other candidate instructive signals. In other words, the learning outcome was best predicted by G-H during training. As the bin size used to calculate mutual information increased, corresponding to lower resolution discrimination of the values of putative instructive signals, the mutual information decreased. Nevertheless, across all bin sizes, the maximum information was carried by G-H. Thus, among all eight candidate instructive signals, the phase of gaze velocity relative to head velocity $(\mathrm{G}-\mathrm{H})$ during training carried the most information about the direction of the learned change in VOR gain (Fig. 4B).

\section{Consistent instructive signals throughout the $30 \mathrm{~min}$ training period}

The analysis above was based on the candidate instructive signals available at the beginning of training, during the first minute of exposure to the visual-vestibular training stimulus. We also measured the phase of gaze relative to head motion $(\mathrm{G}-\mathrm{H})$ at the end of the 30 min training period and found that it was similar to the beginning of training (Fig. 5). The same was true of the other candidate instructive signals considered (data not shown). The similarity of the candidate instructive signals available at the beginning and end of training suggests that the same "learning rules" could operate throughout the entire training period.

\section{Instructive signals for learned changes in VOR phase}

There have been no previous hypotheses about the signals controlling the induction of learned changes in the phase of the VOR, although previous studies have demonstrated that the phase as well as the gain of the VOR can be modified by learning (Shelhamer et al., 1994; Kramer et al., 1995; Katoh et al., 2007, 2008; Andreescu et al., 2011). Therefore, we evaluated whether VOR phase learning may be controlled by the candidate instructive signals we measured.

The training paradigms used in the present experiments induced a range of different learned changes in VOR phase (Fig. $6 A)$. In contrast to the learned change in VOR gain, the learned change in VOR phase could not be consistently predicted from the phase of gaze relative to head motion $(\mathrm{G}-\mathrm{H})$. Values of $\mathrm{G}-\mathrm{H}$ present during training paradigms that induced no learned change in VOR phase (Fig. 6D, black histograms) were highly overlapping with the values present during training paradigms that induced a learned phase lag (gold histograms) or phase lead (green histograms). The values of R-H, R-E, G-H, R-G, $\mathrm{R}_{\mathrm{s}}, \mathrm{E}_{\mathrm{s}}$, or $\mathrm{G}_{\mathrm{s}}$ also failed to consistently predict the learned change in VOR phase (Fig. $6 B, C, F-I$ ). For example, the range of $\mathrm{R}-\mathrm{H}$ values during training paradigms that induced a learned increase in phase lead (Fig. 6B, green shading) encompassed the range of $\mathrm{R}-\mathrm{H}$ values during training paradigms that induced a learned phase lag (Fig. 6, gold histograms) or no change in VOR phase (Fig. 6, black histograms), so that the direction of the learned change in VOR phase could not be predicted from R-H.

The direction of the learned change in VOR phase could be consistently predicted from the phase of the eye movements relative to head motion during training (E-H; Fig. $6 E$ ). E-H values during the training paradigms that induced a learned lead in the VOR phase (Fig. 6E, green shading) were distinct from those during training paradigms that induced a learned phase lag (Fig. $6 E$, yellow shading). E-H values during the training paradigms that induced no change in VOR phase were intermediate between the values associated with learned phase leads and phase lags (Fig. $6 E$, black histograms). A calculation of mutual information confirmed that $\mathrm{E}-\mathrm{H}$ was a better predictor of the learned change in VOR phase than the seven other candidate instructive signals (Fig. 7).

Thus, E-H carried the most information about the direction of the learned phase change (Fig. 7). The values of E-H measured at the end of 30 min training were similar to those measured at the beginning of training (Fig. 8), confirming that E-H provides consistent instructive signals throughout the training period. Therefore, we hypothesize that the "learning rule" controlling VOR phase learning is that a learned phase lead is induced if, during training, eye movements lead head motion in the opposite direction by more than about $30^{\circ}$ (Fig. $6 \mathrm{E}$, green shading), whereas a phase lag is induced if, during training, eye movements lag oppositely directed head motion by more than $\sim 40^{\circ}$ (Fig. $6 E$, yellow shading).

\section{Discussion}

\section{Behavioral instructive signals}

By comparing the VOR learning induced by a broad range of training paradigms, we obtained new constraints on the instructive signals and learning rules guiding this form of cerebellumdependent motor learning. In contrast, if we limited our analysis to the two training paradigms commonly used in laboratories to induce a learned decrease $(\times 0)$ or increase $(\times 2)$ in VOR gain, then all of the learning rules previously proposed to control VOR learning would seem to work. For this limited set of two training conditions, the direction (phase) of retinal slip relative to head motion (R-H), the direction (phase) of retinal slip relative to eye movement (R-E), and the direction (phase) of gaze movement 


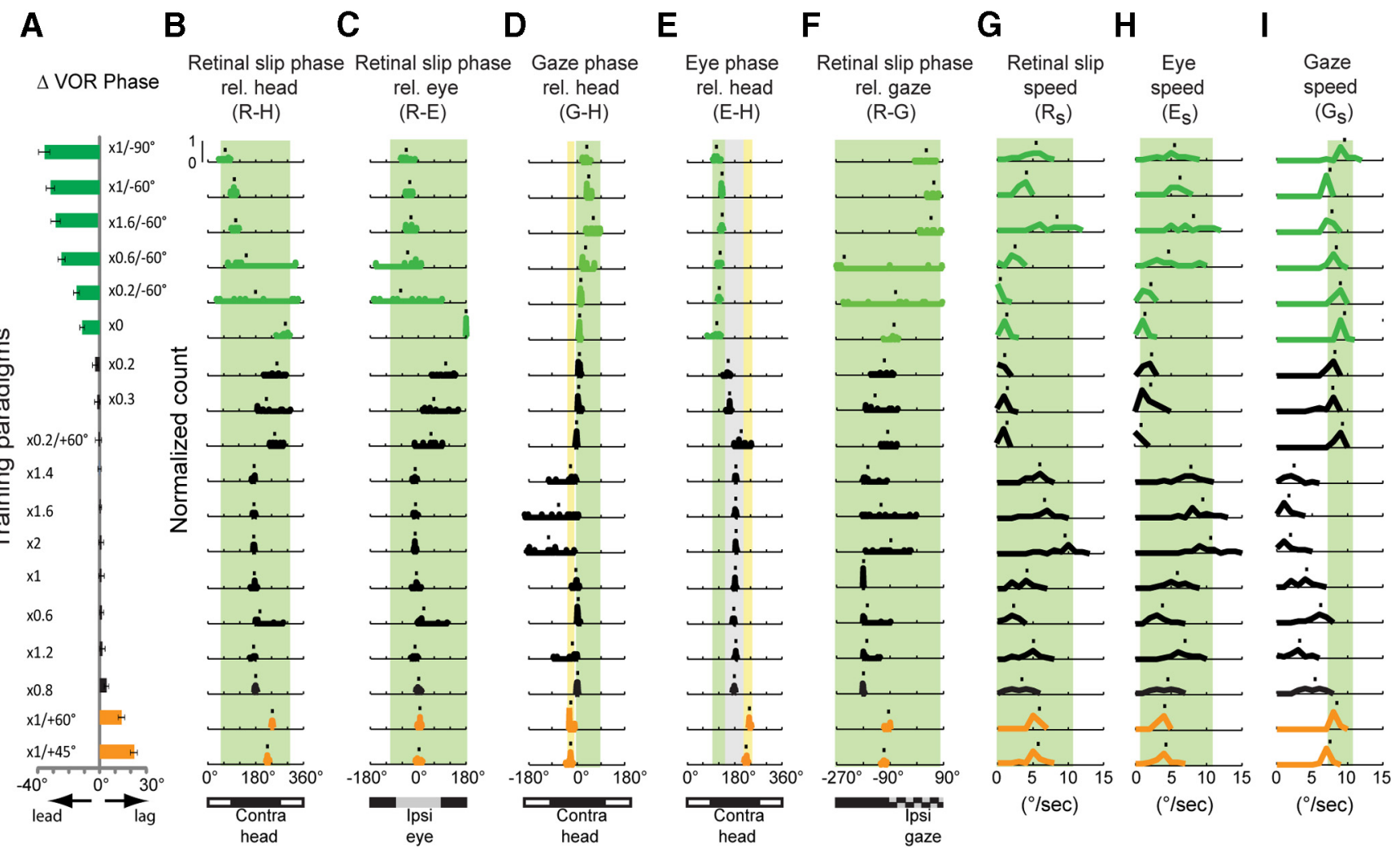

Figure 6. Learned changes in VOR phase induced by the different training paradigms $(\boldsymbol{A})$, and the candidate instructive signals present during training $(\boldsymbol{B}-\boldsymbol{I})$. Green bars and histograms represent results from training paradigms that induced a significant learned lead in VOR phase ( $p<0.05$, Bonferroni-corrected $t$ test). Gold bars and histograms represent training paradigms that induced a learned lag in VOR phase ( $p<0.05$, Bonferroni-corrected $t$ test). Black bars and histograms represent training paradigms that induced no significant change in VOR phase. Green shading represents parameter ranges associated with learned phase lead (mean \pm SEM at the border); yellow shading represents range of E-H values associated with learned phase lag; gray shading represents range of $\mathrm{E}-\mathrm{H}$ values associated with no learned change in VOR phase; no shading represents parameter space not sampled. $\boldsymbol{B}, \boldsymbol{D}, \boldsymbol{E}$, Bottom, Black and white bars represent the alignment with head motion in the opposite direction (black bars, contra-head) or same direction (white bars, ipsi-head), respectively. C, Bottom, Black and gray bars represent the alignment with contraversive (black) or ipsiversive (gray) eye movement. $\boldsymbol{F}$, Bottom, Black and checkerboard bars represent the alignment with contraversive (black) or ipsiversive (checkerboard) gaze movement.

Bin size - Phase $\left({ }^{\circ}\right)$

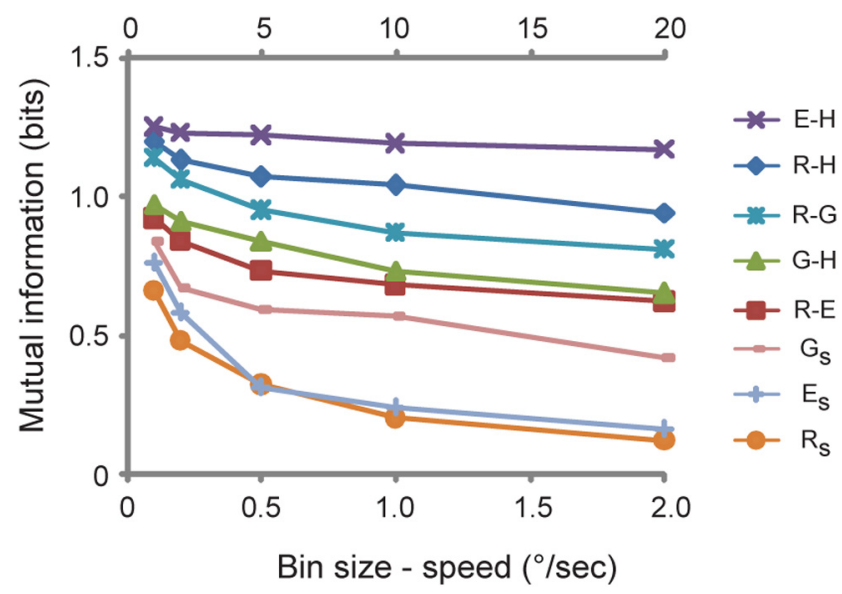

Figure 7. Highest information about learned phase change carried by the phase of eye relative to head (E-H). Mutual information was calculated in either bin size of $1,2,5,10$, or $20^{\circ}$ for $\mathrm{G}-\mathrm{H}, \mathrm{E}-\mathrm{H}, \mathrm{R}-\mathrm{H}$, and $\mathrm{R}-\mathrm{G}$ or bin size of $0.1,0.2,0.5,1$, or $2 \% \mathrm{~s}$ for $\mathrm{R}_{\mathrm{s}^{\prime}} \mathrm{E}_{\mathrm{s}^{\prime}}$ or $\mathrm{G}_{\mathrm{s}}$.

relative to head motion $(\mathrm{G}-\mathrm{H})$ each distinguished the $\times 0$ training paradigm from the $\times 2$ training paradigm and thus seemed to carry the information required to determine the appropriate direction of the learned change in VOR gain (Ito, 1972; Collewijn and Grootendorst, 1979; Mandl et al., 1981; Miles and Lisberger, 1981; Lisberger et al., 1984; Scudder and Fuchs, 1992a; Shelhamer

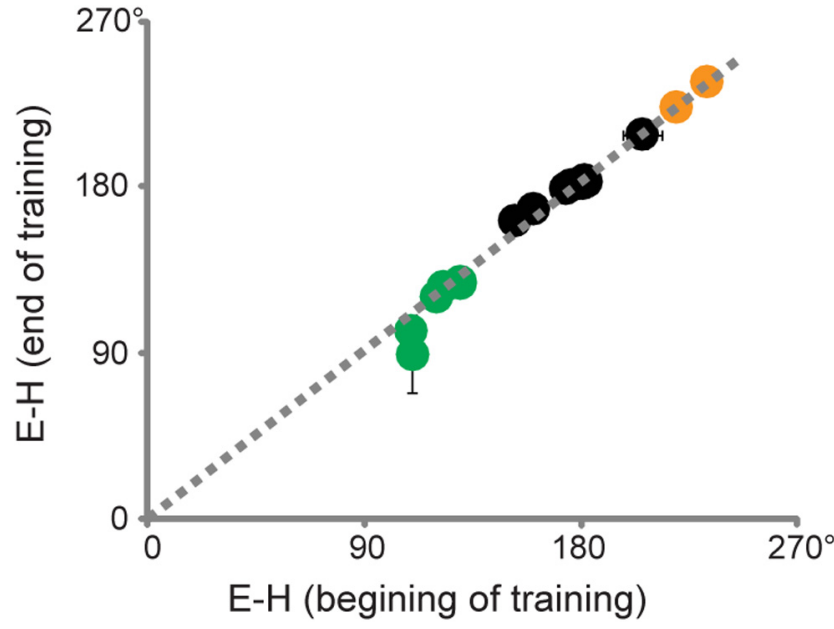

Figure 8. Consistent phase of eye movement relative to head motion (E-H) throughout training period. E-H during the last 5 min of training $(y$-axis) was similar to that during the first 5 min of training ( $x$-axis). Green, black, and gold points represent results from training paradigms that induced a learned lead, no change, or lag in VOR phase, respectively. Dotted line indicates diagonal.

et al., 1994). However, when a broader range of training conditions was examined, only G-H consistently signaled the direction of the learned change in VOR gain.

None of the previous hypotheses about the signals controlling VOR learning could account for the observed changes in VOR 


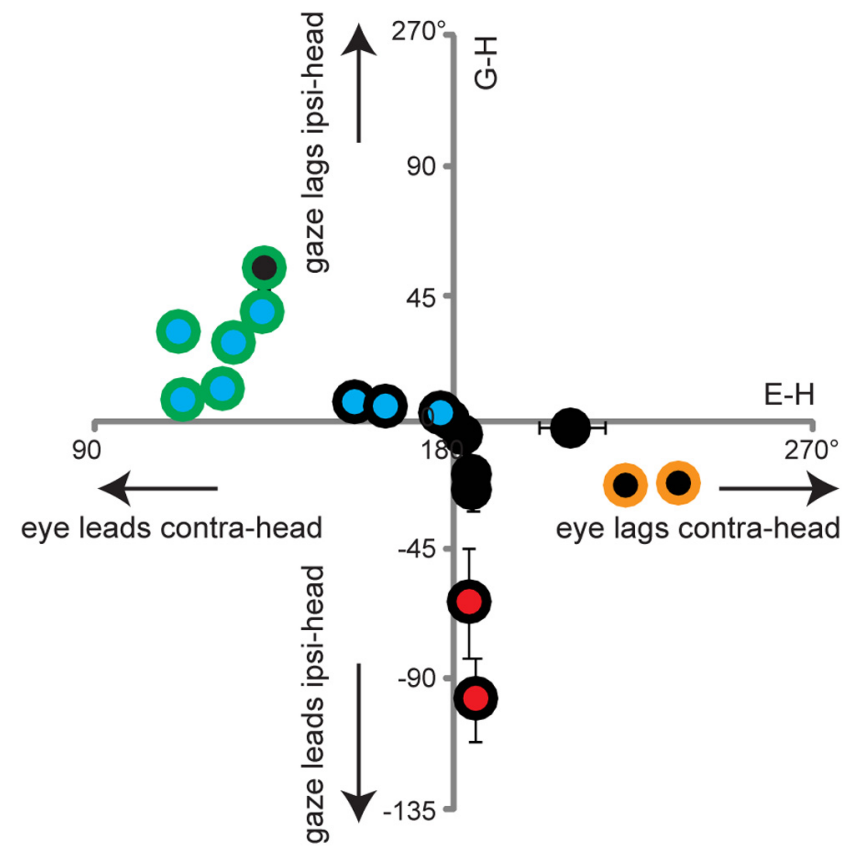

Figure 9. Overlap of the learning rules for learned changes in VOR gain (red, black, or blue filled circles, representing a learned increase, no change, or decrease in VOR gain, respectively) and VOR phase (green, black, or yellow circle outlines, representing a learned lead, no change, or lag in VOR phase, respectively), based on the phase of gaze relative to head motion (G-H, ordinate) and the phase of eye movement relative to head motion (E-H, abscissa).

phase induced by the full set of training paradigms tested. Instead, the direction of the learned change in VOR phase was best predicted by the phase of the eye movement relative to head motion during training (E-H). Thus, different cues seem to control the induction of learned changes in the phase versus the gain of the VOR, consistent with previous reports of different plasticity mechanisms for VOR phase learning versus VOR gain learning (Katoh et al., 2007, 2008). Based on the values of G-H and $\mathrm{E}-\mathrm{H}$ during training, different combinations of gain and phase changes would result (Fig. 9).

It has been suggested that different species might use different instructive signals for VOR learning. In particular, primates might use different signals than other species (Lisberger et al., 1984; Scudder and Fuchs, 1992a; Shelhamer et al., 1994) because similar experiments in primates and rabbits have yielded different hypotheses about the cues controlling VOR learning (Collewijn and Grootendorst, 1979; Miles and Lisberger, 1981). When we use the $\times 0$ and $\times 2$ training protocols in mice that have been used in other species, our results are consistent with the results from other species, and with all of the hypotheses that have been proposed based on the results from other species. It is only when we compare across a broader range of training protocols that we were able to distinguish between the different hypotheses about the cues controlling learning. Thus, it is the use of an expanded range of training protocols rather than the use of mice that supports our new conclusions.

Because primates can use smooth pursuit eye movements to track small targets, which creates retinal slip of background images, retinal slip might provide a less reliable instructive signal for the VOR in primates. Therefore, it has been reasoned that primates might use gaze velocity rather than retinal slip to control VOR learning (Lisberger et al., 1984). Our results suggest that gaze velocity also plays a key role in the induction of VOR learning in nonprimate species, in particu- lar mice, which are not capable of smooth pursuit eye movements. Thus, the instructive signals controlling VOR learning may be shared across species, from rodents to primates.

Our results suggest that learned changes in VOR gain and phase can be induced by the activity of neurons encoding head motion and neurons encoding gaze or eye velocity, respectively. All three signals converge at putative sites of plasticity in the cerebellar flocculi and vestibular nuclei (Dufossé et al., 1978; Miles et al., 1980b; Watanabe, 1984' Lisberger and Pavelko, 1988; Lisberger et al., 1994a; Lisberger et al., 1994b; Lisberger, 1994; Pastor et al., 1994; Partsalis et al., 1995; Menzies et al., 2010). Vestibular signals encoding head motion are conveyed to the vestibular nuclei from the vestibular afferents, and to the cerebellar flocculus via mossy fibers. Eye velocity, which seems to control VOR phase learning, is conveyed to the flocculus by the mossy fibers, is encoded by a subset of Purkinje cells in the flocculus, and also drives firing in some neurons in the vestibular nuclei and possibly the climbing fibers (Noda et al., 1987; Scudder and Fuchs, 1992b; Frens et al., 2001). Gaze velocity, which seems to control VOR gain learning, is encoded by a major subset of Purkinje cells in the flocculus (Miles et al., 1980a, b; Miles and Lisberger, 1981; Lisberger et al., 1994a). It has been proposed that the Purkinje cells drive plasticity downstream in the vestibular nuclei (Miles et al., 1980b; Watanabe, 1984; Lisberger and Pavelko, 1988; Lisberger et al., 1994a, b; Lisberger, 1994; Nguyen-Vu et al., 2013). It is conceivable that the signals carried by these neurons could also influence the induction of plasticity at synapses onto the Purkinje cells.

It is striking that retinal slip was not the best predictor of VOR gain or phase learning. In our experiments, the vestibular stimulus was the same across training conditions, and it was the motion of the visual stimulus that varied and ultimately controlled the learning. Moreover, there is considerable evidence that cerebellar climbing fibers contribute to the induction of plasticity in the cerebellar cortex, and in the flocculus, the climbing fibers encode retinal slip (Ito, 1972; Simpson and Alley, 1974; Barmack and Simpson, 1980; Stone and Lisberger, 1986; Weber et al., 2003; Coesmans et al., 2004; Wu et al., 2010; Nguyen-Vu et al., 2013; Guo et al., 2014; Kimpo et al., 2014). Previous models of VOR learning have proposed that climbing fibers encoding retinal slip signals interact with a mossy fiber input to the cerebellum carrying signals related to head motion (Ito, 1972) or eye movement (Collewijn and Grootendorst, 1979; Porrill and Dean, 2007). However, our results suggest that it was not the retinal slip created by the visual stimulus, but the motor consequence of retinal slip (i.e., eye velocity or gaze velocity) that dominate learning.

It is possible that retinal slip also makes some, albeit more minor, contribution to learning. In primates, manipulation of a visual background can affect VOR gain learning without affecting gaze velocity, suggesting that retinal slip also plays a role in learning (Ke et al., 2009). Thus, more than one set of instructive signals may operate in the VOR circuit to control learning. Our analysis identified the instructive signals that dominate learning across the full set of training conditions tested, but the contributions of different instructive signals may vary across training conditions because there is evidence that different mechanisms mediate different aspects of VOR learning (Kimpo et al., 2005; Boyden et al., 2006; Kimpo et al., 2014). Moreover, more complex combinations of instructive signals than the eight candidate instructive signals tested in the current study may also contribute to learning (Shelhamer et al., 1994). The use of multiple sets of cues to guide 
learning could enable the VOR circuit to respond adaptively to a broader range of conditions.

\section{References}

Andreescu CE, Prestori F, Brandalise F, D’Errico A, De Jeu MT, Rossi P, Botta L, Kohr G, Perin P, D’Angelo E, De Zeeuw CI (2011) NR2A subunit of the $\mathrm{N}$-methyl D-aspartate receptors are required for potentiation at the mossy fiber to granule cell synapse and vestibulo-cerebellar motor learning. Neuroscience 176:274-283. CrossRef Medline

Barmack NH, Simpson JI (1980) Effects of microlesions of dorsal cap of inferior olive of rabbits on optokinetic and vestibuloocular reflexes. J Neurophysiol 43:182-206. Medline

Boyden ES, Raymond JL (2003) Active reversal of motor memories reveals rules governing memory encoding. Neuron 39:1031-1042. CrossRef Medline

Boyden ES, Katoh A, Raymond JL (2004) Cerebellum-dependent learning: the role of multiple plasticity mechanisms. Annu Rev Neurosci 27:581609. CrossRef Medline

Boyden ES, Katoh A, Pyle JL, Chatila TA, Tsien RW, Raymond JL (2006) Selective engagement of plasticity mechanisms for motor memory storage. Neuron 51:823-834. CrossRef Medline

Coesmans M, Weber JT, De Zeeuw CI, Hansel C (2004) Bidirectional parallel fiber plasticity in the cerebellum under climbing fiber control. Neuron 44:691-700. CrossRef Medline

Collewijn H, Grootendorst AF (1979) Adaptation of optokinetic and vestibulo-ocular reflexes to modified visual input in the rabbit. Prog Brain Res 50:771-781. CrossRef Medline

Dufossé M, Ito M, Jastreboff PJ, Miyashita Y (1978) A neuronal correlate in rabbit's cerebellum to adaptive modification of the vestibulo-ocular reflex. Brain Res 150:611-616. CrossRef Medline

Frens MA, Mathoera AL, van der Steen J (2001) Floccular complex spike response to transparent retinal slip. Neuron 30:795-801. CrossRef Medline

Gonshor A, Jones GM (1976) Extreme vestibulo-ocular adaptation induced by prolonged optical reversal of vision. J Physiol 256:381-414. Medline

Guo CC, Ke MC, Raymond JL (2014) Cerebellar encoding of multiple candidate error cues in the service of motor learning. J Neurosci, in press.

Ito M (1972) Neural design of the cerebellar motor control system. Brain Res 40:81-84. CrossRef Medline

Ito M, Shiida T, Yagi N, Yamamoto M (1974) Visual influence on rabbit horizontal vestibulo-ocular reflex presumably effected via the cerebellar flocculus. Brain Res 65:170-174. CrossRef Medline

Katoh A, Jindal JA, Raymond JL (2007) Motor deficits in homozygous and heterozygous p/q-type calcium channel mutants. J Neurophysiol 97: 1280-1287. Medline

Katoh A, Chapman PJ, Raymond JL (2008) Disruption of learned timing in P/Q calcium channel mutants. PLoS One 3:e3635. CrossRef Medline

Ke MC, Guo CC, Raymond JL (2009) Elimination of climbing fiber instructive signals during motor learning. Nat Neurosci 12:1171-1179. CrossRef Medline

Kimpo RR, Boyden ES, Katoh A, Ke MC, Raymond JL (2005) Distinct patterns of stimulus generalization of increases and decreases in VOR gain. J Neurophysiol 94:3092-3100. CrossRef Medline

Kimpo RR, Rinaldi JM, Kim CK, Payne HL, Raymond JL (2014) Gating of neural error signals during motor learning. Elife 3:e02076. CrossRef Medline

Kramer PD, Shelhamer M, Zee DS (1995) Short-term adaptation of the phase of the vestibulo-ocular reflex (VOR) in normal human subjects. Exp Brain Res 106:318-326. Medline

Lisberger SG (1994) Neural basis for motor learning in the vestibuloocular reflex of primates: III. Computational and behavioral analysis of the sites of learning. J Neurophysiol 72:974-998. Medline

Lisberger SG, Pavelko TA (1986) Vestibular signals carried by pathways subserving plasticity of the vestibulo-ocular reflex in monkeys. J Neurosci 6:346-354. Medline

Lisberger SG, Pavelko TA (1988) Brain stem neurons in modified pathways for motor learning in the primate vestibulo-ocular reflex. Science 242: 771-773. CrossRef Medline
Lisberger SG, Miles FA, Zee DS (1984) Signals used to compute errors in monkey vestibuloocular reflex: possible role of flocculus. J Neurophysiol 52:1140-1153. Medline

Lisberger SG, Pavelko TA, Bronte-Stewart HM, Stone LS (1994a) Neural basis for motor learning in the vestibuloocular reflex of primates: II. Changes in the responses of horizontal gaze velocity Purkinje cells in the cerebellar flocculus and ventral paraflocculus. J Neurophysiol 72:954973. Medline

Lisberger SG, Pavelko TA, Broussard DM (1994b) Neural basis for motor learning in the vestibuloocular reflex of primates: I. Changes in the responses of brain stem neurons. J Neurophysiol 72:928-953. Medline

Mandl G, Melvill Jones G, Cynader M (1981) Adaptability of the vestibuloocular reflex to vision reversal in strobe reared cats. Brain Res 209:35-45. CrossRef Medline

Menzies JR, Porrill J, Dutia M, Dean P (2010) Synaptic plasticity in medial vestibular nucleus neurons: comparison with computational requirements of VOR adaptation. PLoS One 5:e13182. CrossRef Medline

Miles FA, Fuller JH (1974) Adaptive plasticity in the vestibulo-ocular responses of the rhesus monkey. Brain Res 80:512-516. Medline

Miles FA, Lisberger SG (1981) Plasticity in the vestibulo-ocular reflex: a new hypothesis. Annu Rev Neurosci 4:273-299. CrossRef Medline

Miles FA, Braitman DJ, Dow BM (1980a) Long-term adaptive changes in primate vestibuloocular reflex: IV. Electrophysiological observations in flocculus of adapted monkeys. J Neurophysiol 43:1477-1493. Medline

Miles FA, Fuller JH, Braitman DJ, Dow BM (1980b) Long-term adaptive changes in primate vestibuloocular reflex: III. Electrophysiological observations in flocculus of normal monkeys. J Neurophysiol 43:1437-1476. Medline

Nguyen-Vu TD, Kimpo RR, Rinaldi JM, Kohli A, Zeng H, Deisseroth K, Raymond JL (2013) Cerebellar Purkinje cell activity drives motor learning. Nat Neurosci 16:1734-1736. CrossRef Medline

Noda H, Warabi T, Ohno M (1987) Response properties and visual receptive fields of climbing and mossy fibers terminating in the flocculus of the monkey. Exp Neurol 95:455-471. CrossRef Medline

Partsalis AM, Zhang Y, Highstein SM (1995) Dorsal Y group in the squirrel monkey: II. Contribution of the cerebellar flocculus to neuronal responses in normal and adapted animals. J Neurophysiol 73:632-650. Medline

Pastor AM, de la Cruz RR, Baker R (1994) Cerebellar role in adaptation of the goldfish vestibuloocular reflex. J Neurophysiol 72:1383-1394. Medline

Porrill J, Dean P (2007) Cerebellar motor learning: when is cortical plasticity not enough? PLoS Comput Biol 3:1935-1950. CrossRef Medline

Robinson DA (1976) Adaptive gain control of vestibuloocular reflex by the cerebellum. J Neurophysiol 39:954-969. Medline

Scudder CA, Fuchs AF (1992a) The error signal for modification of vestibuloocular reflex gain. Ann N Y Acad Sci 656:884-885. CrossRef Medline

Scudder CA, Fuchs AF (1992b) Physiological and behavioral identification of vestibular nucleus neurons mediating the horizontal vestibuloocular reflex in trained rhesus monkeys. J Neurophysiol 68:244-264. Medline

Shelhamer M, Tiliket C, Roberts D, Kramer PD, Zee DS (1994) Short-term vestibulo-ocular reflex adaptation in humans: II. Error signals. Exp Brain Res 100:328-336. Medline

Simpson JI, Alley KE (1974) Visual climbing fiber input to rabbit vestibulocerebellum: a source of direction-specific information. Brain Res 82:302308. CrossRef Medline

Stone LS, Lisberger SG (1986) Detection of tracking errors by visual climbing fiber inputs to monkey cerebellar flocculus during pursuit eye movements. Neurosci Lett 72:163-168. CrossRef Medline

Watanabe E (1984) Neuronal events correlated with long-term adaptation of the horizontal vestibulo-ocular reflex in the primate flocculus. Brain Res 297:169-174. CrossRef Medline

Weber JT, De Zeeuw CI, Linden DJ, Hansel C (2003) Long-term depression of climbing fiber-evoked calcium transients in Purkinje cell dendrites. Proc Natl Acad Sci U S A 100:2878-2883. CrossRef Medline

Wu X, Nestrasil I, Ashe J, Tuite P, Bushara K (2010) Inferior olive response to passive tactile and visual stimulation with variable interstimulus intervals. Cerebellum 9:598-602. CrossRef Medline 